\title{
Combinatorial classification of piecewise hereditary algebras
}

\author{
Marcelo Lanzilotta, Maria Julia Redondo and Rachel Taillefer
}

Keywords: piecewise hereditary algebra; Coxeter polynomial.

Mathematics Subject Classification 2000: 16E60, 15A15, $16 \mathrm{~S} 99$.

\begin{abstract}
We use the characteristic polynomial of the Coxeter matrix of an algebra to complete the combinatorial classification of piecewise hereditary algebras which Happel gave in terms of the trace of the Coxeter matrix. We also give a cohomological interpretation of the coefficients (other than the trace) of the characteristic polynomial of the Coxeter matrix of any finite dimensional algebra with finite global dimension.
\end{abstract}

\section{Introduction}

The main aim of this note is to complete Happel's combinatorial classification of piecewise hereditary algebras.

An algebra $A$ is said to be piecewise hereditary of type $\mathcal{H}$ if its bounded derived category is triangle equivalent to the bounded derived category of a hereditary abelian $k$-category $\mathcal{H}$ for a field $k$ which we assume to be algebraically closed. Such algebras have been much studied, see for instance [5, 6, 9, 10, 11, 12, 13] among others.

Happel and Reiten [9] have shown that $\mathcal{H}$ has a tilting object and therefore $\mathcal{D}^{b}(\mathcal{H})$ is triangle equivalent to $\mathcal{D}^{b}(\Lambda)$ where $\Lambda$ is a finite dimensional hereditary algebra (ie. a path algebra $k \vec{\Delta}$ for a quiver $\vec{\Delta}$ ) or a canonical algebra (see [6]). In the first case we say that $A$ is of type $k \vec{\Delta}$ and in the second case we say that $A$ is of canonical type.

Recall that a canonical algebra is a one-point extension (see Definition 3.3) of the path algebra of the quiver

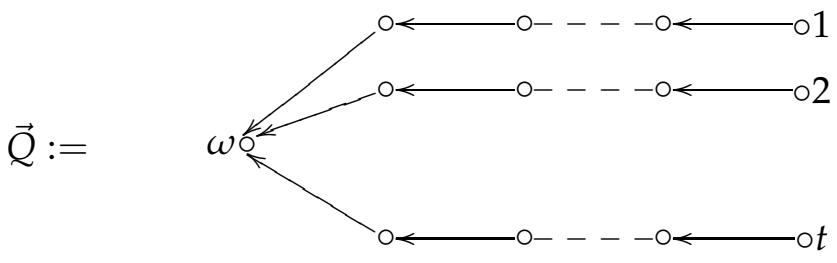

by an indecomposable module $M$ given by the following representation

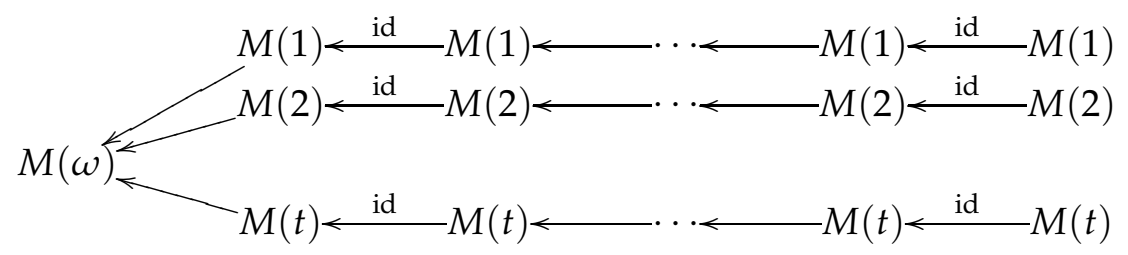

such that $M(1), \ldots, M(t)$ are pairwise different one-dimensional subspaces of $M(\omega), \operatorname{dim}_{k} M(\omega)=2$ and $t \geqslant 2$. If we order the vertices from left to right and from top to bottom, the last vertex being $\omega$, then $M$ has dimension vector $m:=\operatorname{dim}_{k \vec{Q}}(M)=(1, \ldots, 1,2)$ 
Happel has given the following combinatorial characterisation of some piecewise hereditary algebras, using the trace of the Coxeter matrix $\phi_{A}$ of $A$ (see Section 2 for a definition), whose proof follows from [5] and Happel's trace formula [4].

Theorem 1.1 (Happel). Let A be a connected piecewise hereditary algebra over an algebraically closed field. Then

(1) $A$ is of type $k \vec{\Delta}$ where the underlying graph $\Delta$ is not a tree if and only if $\operatorname{tr} \phi_{A}>-1$,

(2) $A$ is of canonical type with $t>3$ branches if and only if $\operatorname{tr} \phi_{A}<-1$,

(3) $A$ is of canonical type with $t=3$ branches or of type $k \vec{\Delta}$ where the underlying graph $\Delta$ is a tree if and only if $\operatorname{tr} \phi_{A}=-1$.

In this paper, we complete this classification, that is, separate path algebra and canonical types when the trace of the Coxeter matrix is -1 :

Theorem 1.2. Let $A$ be a connected piecewise hereditary algebra over an algebraically closed field with $n+1$ isomorphism classes of indecomposable projective modules and $\operatorname{tr} \phi_{A}=-1$. Let $\chi_{A}(x)=\sum_{i=0}^{n+1} \lambda_{i}^{A} x^{i}$ be the characteristic polynomial of the Coxeter matrix $\phi_{A}$ of $A$. Then $A$ is of canonical type (with $t=3$ branches) if and only if one of the three following sets of conditions holds:

(i) $\lambda_{n-1}^{A}=0, \lambda_{n-2}^{A}=-1=\lambda_{n-3}^{A}$ and $\lambda_{n-4}^{A}=0$.

(ii) $\lambda_{n-1}^{A}=0=\lambda_{n-2}^{A}$ and $\lambda_{n-\ell}^{A} \leqslant-1$ for some $\ell \geqslant 3$.

(iii) $\lambda_{n-1}^{A}=1$ and $\lambda_{n-\ell}^{A} \leqslant 0$ for some $\ell \geqslant 2$.

The proof of this theorem is the object of Section 3 .

Finally, in Section 4, we give a cohomological interpretation of the coefficients of the characteristic polynomial of $\phi_{A}$ thus extending Happel's trace formula [4].

\section{Preliminaries}

Assume throughout that $k$ is an algebraically closed field and that all algebras are finite dimensional, basic, connected $k$-algebras and have finite global dimension.

Let $A$ be an algebra and let $P(1), \ldots, P(n)$ be a set of representatives of the isomorphism classes of indecomposable projective left $A$-modules. For a finite dimensional left $A$-module $M$ we denote by $\operatorname{dim}_{A}(M) \in \mathbb{Z}^{n}$ the dimension vector of $M$ : its $i$ th component is $\operatorname{dim}_{k} \operatorname{Hom}_{A}(P(i), M)$. Let $C_{A}$ denote the Cartan matrix of $A$, that is, the $n \times n$ matrix whose $j$ th column is the transpose of $\operatorname{dim}_{A} P(j)$. Since $A$ has finite global dimension, it is well known that $C_{A}$ is invertible over $\mathbb{Z}$. We may therefore consider the following:

- The Coxeter matrix $\phi_{A}=-C_{A}^{-t} C_{A}$ of $A$.

- The Euler form associated to $A,\langle-,-\rangle_{A}: \mathbb{Z}^{n} \times \mathbb{Z}^{n} \rightarrow \mathbb{Z}$ defined by $\langle x, y\rangle_{A}=x C_{A}^{-t} y$. It is known that for two finite dimensional $A$-modules $X$ and $Y$ such that $X$ has finite projective dimension or $Y$ has finite injective dimension we have $\left\langle\operatorname{dim}_{A} X, \operatorname{dim}_{A} Y\right\rangle_{A}=\sum_{i \geqslant 0} \operatorname{dim}_{k} \operatorname{Ext}_{A}^{i}(X, Y)$ (see [16]).

Our main object of study is the Coxeter polynomial of $A$, that is, the characteristic polynomial $\chi_{A}(x)=\operatorname{det}\left(x I_{n}-\phi_{A}\right)$ of the Coxeter matrix of $A$ and in particular its coefficients. The first non-trivial coefficient, that is, the trace, was studied by Happel in [4], who proved that $\operatorname{tr} \phi_{A}=-\left\langle\operatorname{dim}_{A^{e}} A, \operatorname{dim}_{A^{e}} A\right\rangle$ where $A^{e}=A \otimes_{k} A^{o p}$ is the enveloping algebra of $A$.

We are interested in this paper in some of the other coefficients of $\chi_{A}$. 


\section{Classification of piecewise hereditary algebras}

The aim of this section is to prove Theorem 1.2. We begin with a few comments and results that are used in the proof.

Remark 3.1. Let $\mathscr{C}$ be a canonical algebra with $t$ branches. If $p_{i}-1>0$ is the number of vertices in the $i$ th branch, then the sign of $\delta_{p}:=t-2-\sum_{i=1}^{t} \frac{1}{p_{i}}$ gives us information on the representation type of $\mathscr{C}$ (see [2], in particular Remark 5.4 and Proposition 5.5): if $\delta_{p}<0$ then $\mathscr{C}$ is of domestic type and is derived equivalent to the module category of a tame hereditary algebra (in fact for $t=2$, in which case $\delta_{p}<0$, the algebra $\mathscr{C}$ is hereditary by definition), if $\delta_{p}=0$ then $\mathscr{C}$ is of tubular type (studied by Ringel in [16]) and if $\delta_{p}>0$ then $\mathscr{C}$ is of wild type.

Therefore, in order to distinguish piecewise hereditary algebras of canonical type which are not of hereditary type, we need only consider the case $\delta_{p} \geqslant 0$; necessarily we then have $t \geqslant 3$. The case $t>3$ is characterised by the trace (Theorem 1.1), so we may now assume that $t=3$. In this case, $\sum_{i=1}^{3} \frac{1}{p_{i}} \leqslant 1$.

Notation 3.2. When $t=3$, we shall denote the quiver $\vec{Q}$ in the introduction by $\mathbb{T}_{a, b, c}$ where $a=p_{1}-1 \leqslant$ $b=p_{2}-1 \leqslant c=p_{3}-1$ are the number of vertices on each branch in increasing order.

The aim of this section is to separate canonical type from type $k \vec{\Delta}$ when the trace is equal to -1 . For this we shall use an inductive formula for the Coxeter polynomial, which gives the Coxeter polynomial of a one-point extension in terms of that of the original algebra. This was recently proved by Happel in [7]. Recall:

Definition 3.3. Let $B$ be a finite dimensional algebra and let $M$ be a $B$-module. The one-point extension of $B$ by $M$ is the algebra $B[M]=\left(\begin{array}{cc}B & M \\ 0 & k\end{array}\right)$ (with usual matrix addition and multiplication).

The quiver of $B[M]$ contains the quiver of $B$ as a full subquiver and there is an additional vertex, the extension vertex.

Note that when the global dimension of $B$ is finite, so is the global dimension of $B[M]$, so that no further restrictions on $B[M]$ are required.

Let $m$ denote the dimension vector of the $B$-module $M$, i.e. $m=\operatorname{dim}_{B} M$. Then the Cartan matrix of $B[M]$ is $\left(\begin{array}{cc}1 & 0 \\ m^{t} & C_{B}\end{array}\right)$ (if the extension vertex has index 0 so is placed "before" the others) and the Coxeter matrix of $B[M]$ is $\left(\begin{array}{cc}\langle m, m\rangle-1 & -m \phi_{B} \\ -C_{B}^{-t} m^{t} & \phi_{B}\end{array}\right)$ where $\langle-,-\rangle=\langle-,-\rangle_{B}$ is the Euler form for $B$ to simplify notation.

Assume now that $A=B[M]$ is a one-point extension. Let $n$ be the number of isomorphism classes of indecomposable projectives for $B$, so that the number of isomorphism classes of indecomposable projectives for $A$ is $n+1$. Denote by $\chi_{A}(x)=\sum_{i=0}^{n+1} \lambda_{i}^{A} x^{i}$ the Coxeter polynomial of $A$ and by $\chi_{B}(x)=\sum_{i=0}^{n} \lambda_{i}^{B} x^{i}$ the Coxeter polynomial of $B$.

Theorem 3.4. [7. Theorem 2.1] With the notation above, for any integer $\ell$ with $0 \leqslant \ell \leqslant n$, we have

$$
\lambda_{n+1-\ell}^{A}=\lambda_{n-\ell}^{B}-(\langle m, m\rangle-1) \lambda_{n-(\ell-1)}^{B}-\sum_{i=1}^{\ell-1} \lambda_{n-\ell+i+1}^{B}\left\langle m \phi_{B}^{i}, m\right\rangle .
$$

The rest of the section is devoted to the proof of Theorem 1.2 and relies on several lemmas which we give now.

Note that since the Coxeter polynomial is a derived invariant (see eg. [7]), we need only consider the Coxeter polynomials of path algebras of trees and of canonical algebras with three branches. Moreover, let $\vec{\Delta}_{1}$ and $\vec{\Delta}_{2}$ be two quivers with the same underlying tree graph $\Delta$. Let $A_{1}=k \vec{\Delta}_{1}$ and $A_{2}=k \vec{\Delta}_{2}$ be 
the corresponding path algebras. It is known by eg. [8] and [3] that $A_{1}$ and $A_{2}$ are derived equivalent. Therefore the Coxeter polynomials of $A_{1}$ and $A_{2}$ are equal. We shall also denote them by $\chi_{\Delta}$.

The Coxeter polynomial of $k \overrightarrow{\mathbb{A}}_{n+1}$ for the Dynkin graph $\mathbb{A}_{n+1}$ is well known, it is equal to $\sum_{i=0}^{n+1} x^{i}$ (see eg. [7, 17, 1]).

Lemma 3.5. Consider the path algebra $k \overrightarrow{\mathbb{T}}_{a, b, c}$ with $a \leqslant b \leqslant c$. Then

$$
\lambda_{(a+b+c)-\ell}^{k \overrightarrow{\mathbb{T}}_{a, b, c}}=\frac{(1-\ell)(2+\ell)}{2} \quad \text { for } 0 \leqslant \ell \leqslant a .
$$

Moreover, if $a=1$ then $\chi_{\mathbb{T}_{1, b, c}}(x)=x^{b+c+2}+\sum_{j=c+1}^{b+c+1}(j-b-c) x^{j}+\sum_{j=b+1}^{c}(1-b) x^{j}+\sum_{j=1}^{b}(2-j) x^{j}+1$. In particular, for the Dynkin graph $\mathbb{D}_{n+1}=\mathbb{T}_{1,1, n-2}$ we get $\chi_{\mathbb{D}_{n+1}}(x)=x^{n+1}+x^{n}+x+1$.

Remark 3.6. Note that the Coxeter polynomial of $\mathbb{D}_{n+1}$ has been computed elsewhere, see for instance [7, 17]. Moreover, this lemma contains the cases of the Dynkin graphs $\mathbb{E}$ and of the Euclidean graphs $\widetilde{\mathbb{E}}$ which can also be found for instance in [7, 17].

Proof. To compute the Coxeter polynomial of $k \overrightarrow{\mathbb{T}}_{a, b, c}$, we use a result of Boldt [1, Corollary 3.2] (see also [17]) which gives

$$
\chi_{\mathbb{T}_{a, b, c}}(x)=\left(\sum_{i=0}^{a} x^{i}\right)\left(\sum_{i=0}^{b+c+1} x^{i}\right)-x\left(\sum_{i=0}^{a-1} x^{i}\right)\left(\sum_{i=0}^{b} x^{i}\right)\left(\sum_{i=0}^{c} x^{i}\right)
$$

(since the Coxeter polynomials for the $\mathbb{A}_{\ell}$ are known). It is easy to check that

$$
\left(\sum_{i=0}^{a} x^{i}\right)\left(\sum_{i=0}^{b+c+1} x^{i}\right)=\sum_{p=0}^{a}(p+1) x^{p}+\sum_{p=a+1}^{b+c+1}(a+1) x^{p}+\sum_{p=b+c+2}^{a+b+c+1}(a+b+c+1-p+1) x^{p} .
$$

Similarly,

$$
x\left(\sum_{i=0}^{a-1} x^{i}\right)\left(\sum_{i=0}^{b} x^{i}\right)\left(\sum_{i=0}^{c} x^{i}\right)=\left(\sum_{i=1}^{a} x^{i}\right)\left(\sum_{q=0}^{b-1}(q+1) x^{q}+\sum_{q=b}^{c}(b+1) x^{q}+\sum_{q=c+1}^{b+c}(b+c-q+1) x^{q}\right)
$$

in which the coefficient of $x^{a+b+c-\ell}$ is

$$
\begin{cases}\sum_{q=b+c-\ell}^{b+c}(b+c-q+1)=\frac{(\ell+1)(\ell+2)}{2} & \text { if } 0 \leqslant \ell \leqslant a-1 \\ \sum_{q=b+c-a}^{b+c-1}(b+c-q+1)=\frac{a(a+3)}{2} & \text { if } \ell=a .\end{cases}
$$

Finally, the coefficient of $x^{p}$ in $\chi_{\mathbb{T}_{a, b, c}}(x)$ is $-\frac{(a+b+c-1-p)(a+b+c+2-p)}{2}$ if $b+c \leqslant p \leqslant a+b+c$ as required.

Lemma 3.7. Let $\Delta$ be a tree with $n+1$ vertices which is neither $\mathbb{A}_{n+1}$ nor $\mathbb{T}_{a, b, c}$. Then $\lambda_{n-1}^{\Delta} \leqslant-1$.

Remark 3.8. This lemma contains the Euclidean cases $\widetilde{\mathbb{D}}_{n}$ whose Coxeter polynomials are known entirely (see [7, 17]).

Proof. $\Delta$ is characterised by the fact that it has either two or more vertices of valency 3 (the valency of a vertex is the number of edges connected to it) or at least one vertex of valency at least 4 . Applying [7, Theorem 4.8] gives $\lambda_{n-1}^{\Delta} \leqslant-1$ in all these cases. 
Let $A=\mathscr{C}_{a, b, c}$ denote the canonical algebra which is a one-point extension $B[M]$ of the tree $B=k \mathbb{T}_{a, b, c}$ (with $a \leqslant b \leqslant c$ ) as defined in the introduction. Recall that we need only consider the cases where $\frac{1}{a+1}+\frac{1}{b+1}+\frac{1}{c+1} \leqslant 1$. We have $m:=\operatorname{dim}_{B}(M)=(1, \ldots, 1,2)$.

We shall use Theorem 3.4 to compute coefficients of the Coxeter polynomial of $A$. We always have $\lambda_{n+1}^{A}=1, \lambda_{n}^{A}=-\operatorname{tr} \phi_{A}=1$.

We first need the Coxeter matrix of $B$; this can be determined using [1, Proposition 3.1]. Before we give it, we introduce some notation; for positive integers $p, q$ we set:

- $J_{p}=\left(\begin{array}{cc}0 & 0 \\ I_{p-1} & 0\end{array}\right)\left(\right.$ a $p \times p$ matrix; $I_{p-1}$ denotes the identity matrix $)$

- $K_{p, q}=\left(\begin{array}{ccc}1 & \cdots & 1 \\ 0 & \cdots & 0 \\ \vdots & \cdots & \vdots \\ 0 & \cdots & 0\end{array}\right)($ a $p \times q$ matrix $)$

- $\varepsilon_{p}^{(q)}$ the $p$ th vector in the canonical basis of $k^{q}(p \leqslant q)$

- $v_{q}=\sum_{p=1}^{q} \varepsilon_{p}^{(q)}=(1, \ldots, 1)$.

They satisfy the following rules: for any positive integers $p, q, r$ we have

$$
\begin{array}{ll}
v_{q} J_{q}=v_{q}-\varepsilon_{q}^{(q)} & v_{p} K_{p, q}=v_{q} \\
\varepsilon_{p}^{(q)} J_{q}= \begin{cases}\varepsilon_{p-1}^{(q)} \text { if } p \geqslant 2 & \varepsilon_{p}^{(q)} K_{q, r}=\left\{\begin{array}{l}
0 \text { if } p \geqslant 2 \\
0 \text { if } p=1
\end{array} v_{r} \text { if } p=1 .\right.\end{cases}
\end{array}
$$

With this notation, we have $m=\left(v_{a}, v_{b}, v_{c}, 2 v_{1}\right)$. Using [1, Proposition 3.1] we have

$$
\phi_{B}=\left(\begin{array}{cccc}
J_{a} & K_{a, b} & K_{a, c} & K_{a, 1} \\
K_{b, a} & J_{b} & K_{b, c} & K_{b, 1} \\
K_{c, a} & K_{c, b} & J_{c} & K_{c, 1} \\
-K_{1, a} & -K_{1, b} & -K_{1, c} & -1
\end{array}\right) .
$$

Moreover, for all $i \geqslant 0$ we have $\left\langle m \phi_{B}^{i}, m\right\rangle=\left\langle m \phi_{B}^{i}, m\right\rangle^{t}=m C_{B}^{-1}\left(m \phi_{B}^{i}\right)^{t}$ and Boldt explains in [1] how to compute $C_{B}^{-1}$; we get

$$
m C_{B}^{-1}=\left(-\varepsilon_{1}^{(a)},-\varepsilon_{1}^{(b)},-\varepsilon_{1}^{(c)}, 2 \varepsilon_{1}^{(1)}\right) .
$$

We always have $\langle m, m\rangle=1$.

Lemma 3.9. Let $A$ be the canonical algebra $\mathscr{C}_{1,2, c}$ with $c \geqslant 5$. Then $\lambda_{n-1}^{A}=0, \lambda_{n-2}^{A}=-1=\lambda_{n-3}^{A}$ and $\lambda_{n-4}^{A}=0$.

Proof. We have $m \phi_{B}=\left(0, \varepsilon_{1}^{(2)}, v_{c}-\varepsilon_{c}^{(c)}, v_{1}\right), m \phi_{B}^{2}=\left(v_{1}, 0, v_{c}-\varepsilon_{c}^{(c)}-\varepsilon_{c-1}^{(c)}, v_{1}\right), m \phi_{B}^{3}=\left(0, v_{2}, v_{c}-\varepsilon_{c}^{(c)}-\right.$ $\left.\varepsilon_{c-1}^{(c)}-\varepsilon_{c-2}^{(c)}, v_{1}\right)$ and $m \phi_{B}^{4}=\left(v_{1}, v_{2}-\varepsilon_{2}^{(2)}, v_{c}-\varepsilon_{c}^{(c)}-\varepsilon_{c-1}^{(c)}-\varepsilon_{c-2}^{(c)}-\varepsilon_{c-3}^{(c)}, v_{1}\right)$, so that $\left\langle m \phi_{B}^{i}, m\right\rangle=0$ for $1 \leqslant i \leqslant 3$ and $\left\langle m \phi_{B}^{4}, m\right\rangle=-1$. We know the coefficients of $\chi_{B}(x)$ from Lemma $3.5, \lambda_{n}^{B}=1, \lambda_{n-1}^{B}=1$, $\lambda_{n-2}^{B}=0, \lambda_{n-3}^{B}=-1, \lambda_{n-4}^{B}=-1$ and $\lambda_{n-5}^{B}=-1$. Therefore the formula in Theorem 3.4 gives the result.

Lemma 3.10. Let $A$ be the canonical algebra $\mathscr{C}_{1, b, c}$ with $b \geqslant 3$. Then $\lambda_{n-r}^{A}=0$ for $1 \leqslant r \leqslant b-1$ and $\lambda_{n-b}^{A}<0$.

Proof. We have $m \phi_{B}=\left(0, v_{b}-\varepsilon_{b}^{(b)}, v_{c}-\varepsilon_{c}^{(c)}, v_{1}\right)$ and for $2 \leqslant r \leqslant b$, by induction,

$$
m \phi_{B}^{r}=\left(F_{r-2} v_{1}, F_{r-1} v_{b}-\sum_{k=0}^{r-2} F_{r-2-k} \varepsilon_{b-k}^{(b)}-\varepsilon_{b-r+1}^{(b)}, F_{r-1} v_{c}-\sum_{k=0}^{r-2} F_{r-2-k} \varepsilon_{c-k}^{(c)}-\varepsilon_{c-r+1}^{(c)}, F_{r-1} v_{1}\right)
$$


where $F_{r}$ is the $r$ th term in the Fibonacci sequence $\left(F_{0}=1=F_{1}\right.$ and $F_{r+2}=F_{r+1}+F_{r}$ for $\left.r \geqslant 0\right)$. Therefore $\left\langle m \phi_{B}, m\right\rangle=0,\left\langle m \phi_{B}^{r}, m\right\rangle=-F_{r-2}$ for $2 \leqslant r \leqslant b-1$ and $\left\langle m \phi_{B}^{b}, m\right\rangle=-F_{b-2}+1+\delta_{b c}$. We also know by Lemma 3.5 that $\lambda_{n-i}^{B}=\left\{\begin{array}{l}1 \text { if } i=0 \\ 2-i \text { if } 1 \leqslant i \leqslant b+1\end{array}\right.$ so that by Theorem 3.4 we get

$$
\left\{\begin{array}{l}
\lambda_{n-1}^{A}=0 \\
\lambda_{n-r}^{A}=1-r+\sum_{i=2}^{r-1}(2+i-r) F_{i-2}+F_{r-2} \text { if } 2 \leqslant r \leqslant b-1 \\
\lambda_{n-b}^{A}=-\delta_{b c}-b+\sum_{i=2}^{b-1}(2+i-b) F_{i-2}+F_{b-2} .
\end{array}\right.
$$

We use the well known formula $\sum_{i=p}^{q} F_{i}=F_{q+2}-F_{p+1}$ to get $\sum_{i=0}^{q} i F_{i}=\sum_{k=1}^{q} \sum_{i=k}^{q} F_{i}=q F_{q+2}-F_{q+3}+F_{3}$. These finally give:

$$
\left\{\begin{array}{l}
\lambda_{n-r}^{A}=0 \text { if } 1 \leqslant r \leqslant b-1 \\
\lambda_{n-b}^{A}=-\delta_{b c}-1<0
\end{array}\right.
$$

Lemma 3.11. Let $A$ be the canonical algebra $\mathscr{C}_{a, b, c}$ with $a \geqslant 2$. Then $\lambda_{n-r}^{A}=1$ for $1 \leqslant r \leqslant a-1$ and $\lambda_{n-a}^{A} \leqslant 0$.

Proof. For $1 \leqslant r \leqslant a$, we prove by induction that the vector $m \phi_{B}^{r}$ is equal to

$$
\left(2^{r-1} v_{a}-\sum_{j=0}^{r-2} 2^{r-2-j} \varepsilon_{a-j}^{(a)}-\varepsilon_{a-r+1}^{(a)}, 2^{r-1} v_{b}-\sum_{j=0}^{r-2} 2^{r-2-j} \varepsilon_{b-j}^{(b)}-\varepsilon_{b-r+1}^{(b)}, 2^{r-1} v_{c}-\sum_{j=0}^{r-2} 2^{r-2-j} \varepsilon_{c-j}^{(c)}-\varepsilon_{c-r+1}^{(c)}, 2^{r-1} v_{1}\right)
$$

so that $\left\langle m \phi_{B}^{r}, m\right\rangle=-2^{r-1}$ if $1 \leqslant r \leqslant a-1$ and $\left\langle m \phi_{B}^{a}, m\right\rangle=-2^{a-1}+1+\delta_{a b}+\delta_{a c}$. Then using Theorem 3.4 and the coefficients of $\chi_{B}$ obtained previously in Lemma 3.5 we get

$$
\left\{\begin{array}{l}
\lambda_{n-i}^{A}=1 \text { if } 1 \leqslant i \leqslant a-1 \\
\lambda_{n-a}^{A}=-\delta_{a b}-\delta_{a c} \leqslant 0
\end{array}\right.
$$

(we use the relations $\sum_{\ell=1}^{p} 2^{-\ell}(\ell+1)=3-2^{-p}(p+3)$ and $\sum_{\ell=1}^{p} \ell(\ell+1) 2^{-\ell+1}=2^{4}-2^{1-p}\left(p^{2}+5 p+8\right)$ obtained by differentiating the identity $\sum_{\ell=1}^{p} x^{\ell+1}=x^{2} \frac{x^{p}-1}{x-1}$ twice and evaluating at $\left.x=2^{-1}\right)$.

We now have all we need to finish the proof of the classification.

Proof of Theorem 1.2 Consider the Coxeter polynomial of $A=\mathscr{C}_{a, b, c}$ with $a \geqslant 2$ (and $n=a+b+c+1$ ). Then $\lambda_{n-1}^{A}=1$ and using Lemmas 3.5 and 3.7 we see that the only tree that satisfies this is $\mathbb{A}_{n+1}$. However, the coefficients of $x^{n-a}$ in the Coxeter polynomials of $\mathbb{A}_{n+1}$ and $\mathscr{C}_{a, b, c}$ differ since it is 1 for $\mathbb{A}_{n+1}$ and nonpositive for $\mathscr{C}_{a, b, c}$ by Lemma 3.11. Therefore the Coxeter polynomial of $\mathscr{C}_{a, b, c}$ is different from that of all trees.

Now consider the Coxeter polynomial of $\mathscr{C}_{1,2, c}$ with $c \geqslant 2$ (and $n=c+4$ ). Recall from Remark 3.1 that we need only consider the case where $1 \geqslant \frac{1}{a+1}+\frac{1}{b+1}+\frac{1}{c+1}=\frac{1}{2}+\frac{1}{3}+\frac{1}{c+1}$, ie. $c \geqslant 5$, so that we assume $c \geqslant 5$. Using Lemmas 3.5 and 3.7 we see that the only tree such that the coefficients of $x^{n+1-i}$ for $0 \leqslant i \leqslant 4$ in its Coxeter polynomial are the same as those for $\mathscr{C}_{1,2, c}$ is $\mathbb{T}_{1,2, c+1}$. But the coefficient of $x^{c}$ in the Coxeter polynomial of $\mathbb{T}_{1,2, c+1}$ is -1 whereas for $\mathscr{C}_{1,2, c}$ it is 0 by Lemma 3.9. Therefore the Coxeter polynomial of $\mathscr{C}_{1,2, c}$ is different from that of all trees.

Finally consider the Coxeter polynomial of $\mathscr{C}_{1, b, c}$ with $b \geqslant 3$ (and $n=b+c+2$ ). Using Lemmas 3.5 and 3.7 we see that the only tree such that the coefficients of $x^{n+1-i}$ for $0 \leqslant i \leqslant 3$ in its Coxeter polynomial are the same as those for $\mathscr{C}_{1, b, c}$ is $\mathbb{D}_{b+c+3}$. But the coefficient of $x^{c+2}$ is 0 in the Coxeter polynomial of $\mathbb{D}_{b+c+3}$ and in that of $\mathscr{C}_{1, b, c}$ it is negative by Lemma 3.10. Therefore the Coxeter polynomial of $\mathscr{C}_{1, b, c}$ is different from that of all trees. 


\section{Cohomological interpretation of the coefficients of the Coxeter polyno- mial}

Happel proved the following result:

Theorem 4.1. [4] $\operatorname{tr} \phi_{A}=-\left\langle\operatorname{dim}_{A^{e}} A, \operatorname{dim}_{A^{e}} A\right\rangle$.

We wish to do something similar for the other coefficients of the Coxeter polynomial. From now on, assume that the characteristic of $k$ is 0 . We need an interpretation of these coefficients in terms of the entries of the Coxeter matrix:

Proposition 4.2. Let $\phi=\left(\phi_{i j}\right)_{1 \leqslant i, j \leqslant n}$ be a matrix, and let $\chi(x)=\operatorname{det}(x \mathrm{id}-\phi)$ be its characteristic polynomial. Write $\chi(x)=x^{n}+\lambda_{n-1} x^{n-1}+\cdots+\lambda_{1} x+\lambda_{0}$. Then

$$
\lambda_{n-\ell}=\sum(-1)^{\sigma(\underline{p})} \alpha_{\underline{p}} \operatorname{tr}\left(\phi^{p_{1}}\right) \cdots \operatorname{tr}\left(\phi^{p_{r}}\right),
$$

where the sum is taken over all partitions $\underline{p}=\left(p_{1}, \ldots, p_{r}\right)$ of $\ell, \sigma(\underline{p})=\sum_{i=1}^{r} p_{i}$ and $\alpha_{\underline{p}}=$ $\frac{1}{p_{1} p_{2} \ldots p_{r}} \prod_{a=0}^{\ell} \frac{1}{\left(\#\left\{i \mid p_{i}=a\right\}\right) !}$.

Proof. Without loss of generality, we may assume that the field $k$ is algebraically closed. Therefore the coefficient $\lambda_{n-\ell}$ of the characteristic polynomial of $\phi$ is the $\ell$ th elementary symmetric polynomial $\sigma_{\ell}\left(\mu_{1}, \ldots, \mu_{n}\right)$ in the eigenvalues $\mu_{1}, \ldots, \mu_{n}$ of $\phi$. This can be expressed in terms of the symmetric polynomials $S_{k}=\sum_{i=1}^{n} \mu_{i}^{k}=\operatorname{tr}\left(\phi^{k}\right)$ using Waring's formula, see for instance [14, V.2] or [15, I.6], which gives the expression above.

We now need to introduce some notation and results.

Let $p_{A}(i)\left(\right.$ resp. $q_{A}(i)$, resp. $\left.e_{A}(i)\right)$ denote the dimension vector of the $i$ th indecomposable projective $A$-module $P(i)$ (resp. the indecomposable injective $A$-module $Q(i)$, resp. the $i$ th simple $A$-module $S(i)$ ). Let $e_{1}, \ldots, e_{n}$ be the primitive orthogonal idempotents in $A$ such that $P(i)=A e_{i}$. Let $D$ denote the $k$-dual, ie. $D=\operatorname{Hom}_{k}(-, k)$ and let $\langle-,-\rangle_{A}$ denote the Euler form as before.

We have mentioned before that the transpose of $p_{A}(i)$ is the $i$ th column of the Cartan matrix $C_{A}$. It is known that $q_{A}(i)$ is the $i$ th row of $C_{A}$ (see [16]).

We shall also need to work with bimodules. The indecomposable projective $A^{e}$-modules are the $A e_{i} \otimes e_{j} A$. Set $e_{i, j}=e_{i} \otimes e_{j} \in A^{e}$, and let $S(i, j)$ be the corresponding simple module with $e_{A^{e}}(i, j)$ its dimension vector.

We order the idempotents in the following way:

$$
e_{1,1}, \ldots, e_{n, 1}, e_{1,2}, \ldots, e_{n, 2}, \ldots, e_{1, n}, \ldots, e_{n, n} .
$$

Happel recalled in [4] the following results: $\operatorname{dim}_{A^{e}} A=\left(p_{A}(1), \ldots, p_{A}(n)\right)$, and $C_{A^{e}}^{-t}=C_{A}^{-1} \otimes C_{A}^{-t}$.

The dual $D A$ is an $A^{e}$-module. Its dimension vector is given by:

$$
\begin{aligned}
\operatorname{dim}_{k} \operatorname{Hom}_{A^{e}}\left(A^{e} e_{i, j}, D A\right) & =\operatorname{dim}_{k} \operatorname{Hom}_{A^{e}}\left(A^{e} e_{i, j}, \operatorname{Hom}_{k}(A, k)\right) \\
& =\operatorname{dim}_{k} \operatorname{Hom}_{k}\left(A \otimes_{A^{e}} A^{e} e_{i, j}, k\right) \\
& =\operatorname{dim}_{k} D\left(A \otimes_{A^{e}} A^{e} e_{i, j}\right)=\operatorname{dim}_{k} D\left(A e_{i, j}\right)=\operatorname{dim}_{k} D\left(e_{j} A e_{i}\right) \\
& =\operatorname{dim}_{k}\left(e_{j} A e_{i}\right)=\operatorname{dim}_{k} \operatorname{Hom}_{A}(P(j), P(i))=q_{A}(j)_{i} .
\end{aligned}
$$

Therefore $\operatorname{dim}_{A^{e}} D A=\left(q_{A}(1), \ldots, q_{A}(n)\right)$.

We may now give a cohomological interpretation of the trace of the powers $\phi_{A}$ and hence of the coefficients of the Coxeter polynomial of $A$ : 
Theorem 4.3. Let $D A$ denote the dual of $A$, viewed as a bimodule over $A$. Then

- $\operatorname{tr}\left(\phi_{A}^{2}\right)=\left\langle\operatorname{dim}_{A^{e}} D A, \operatorname{dim}_{A^{e}} A\right\rangle$.

- If $k \geqslant 3$, then $(-1)^{k} \operatorname{tr}\left(\phi_{A}^{k}\right)$ is equal to

$$
\sum_{1 \leqslant v_{1}, \ldots, v_{k-1} \leqslant n}\left\langle q_{A}\left(v_{1}\right), p_{A}\left(v_{k-1}\right)\right\rangle_{A}\left\langle q_{A}\left(v_{2}\right), e_{A}\left(v_{1}\right)\right\rangle_{A} \ldots\left\langle q_{A}\left(v_{k-2}\right), e_{A}\left(v_{k-3}\right)\right\rangle_{A}\left\langle\operatorname{dim}_{A^{e}} D A, e_{A^{e}}\left(v_{k-1}, v_{k-2}\right)\right\rangle_{A^{e}}
$$

Proof. We write $\langle-,-\rangle=\langle-,-\rangle_{A}$ to simplify notation.

- We first prove that if $X \in \mathbb{Z}^{n}$ and $r \in \mathbb{N}, r \geqslant 1$, then

$$
\left(C_{A} C_{A}^{-t}\right)^{r} X^{t}=\sum_{1 \leqslant u_{1}, \ldots, u_{r} \leqslant n}\left\langle q_{A}\left(u_{1}\right), X\right\rangle\left\langle q_{A}\left(u_{2}\right), e_{A}\left(u_{1}\right)\right\rangle \ldots\left\langle q_{A}\left(u_{r}\right), e_{A}\left(u_{r-1}\right)\right\rangle e_{A}\left(u_{r}\right)^{t}=: Y^{t},
$$

by induction on $r$ :

If $r=1$, we have

$$
C_{A} C_{A}^{-t} X^{t}=\left(\begin{array}{c}
q_{A}(1) \\
\vdots \\
q_{A}(n)
\end{array}\right) C_{A}^{-t} X^{t}=\left(\begin{array}{c}
\left\langle q_{A}(1), X\right\rangle \\
\vdots \\
\left\langle q_{A}(n), X\right\rangle
\end{array}\right)=\sum_{u=1}^{n}\left\langle q_{A}(u), X\right\rangle e_{A}(u)^{t} .
$$

Assume the result is true for $r$; then

$$
\begin{aligned}
&\left(C_{A} C_{A}^{-t}\right)^{r+1} X^{t}=C_{A} C_{A}^{-t} Y^{t}=\sum_{u_{r+1}=1}^{n}\left\langle q_{A}\left(u_{r+1}\right), Y\right\rangle e_{A}\left(u_{r+1}\right)^{t} \\
&=\sum_{1 \leqslant u_{1}, \ldots, u_{r}, u_{r+1} \leqslant n}\left\langle q_{A}\left(u_{1}\right), X\right\rangle\left\langle q_{A}\left(u_{2}\right), e_{A}\left(u_{1}\right)\right\rangle \ldots \\
&\left\langle q_{A}\left(u_{r}\right), e_{A}\left(u_{r-1}\right)\right\rangle\left\langle q_{A}\left(u_{r+1}\right), e_{A}\left(u_{r}\right)\right\rangle e_{A}\left(u_{r+1}\right)^{t} .
\end{aligned}
$$

- Set $C_{A}=\left(c_{i j}\right)_{1 \leqslant i, j \leqslant n}$ and $C_{A}^{-1}=\left(\zeta_{i j}\right)_{1 \leqslant i, j \leqslant n}$. Then

$$
\begin{aligned}
\operatorname{tr}\left(\phi_{A}^{2}\right) & =\sum_{1 \leqslant i, j \leqslant n} \phi_{i j} \phi_{j i}=\sum_{1 \leqslant i, j, r, s \leqslant n} \zeta_{r i} c_{r j} \zeta_{s j} c_{s i}=\sum_{i, r} \zeta_{r i} q_{A}(r) C_{A}^{-t} p_{A}(i)^{t} \\
& =\left(q_{A}(1), \ldots, q_{A}(n)\right) C_{A}^{-1} \otimes C_{A}^{-t}\left(\begin{array}{c}
p_{A}(1)^{t} \\
\vdots \\
p_{A}(n)^{t}
\end{array}\right) \\
& =\operatorname{dim}_{A^{e}}(D A) C_{A^{e}}^{-t}\left(\operatorname{dim}_{A^{e}} A\right)^{t}=\left\langle\operatorname{dim}_{A^{e}} D A, \operatorname{dim}_{A^{e}} A\right\rangle .
\end{aligned}
$$

- Assume $k \geqslant 3$. Then

$$
\begin{aligned}
& \operatorname{tr}\left(\phi_{A}^{k}\right)= \sum_{1 \leqslant j_{1}, \ldots, j_{k} \leqslant n} \phi_{j_{1} j_{2}} \phi_{j_{2} j_{3}} \ldots \phi_{j_{k} j_{1}}=\sum_{\substack{1 \leqslant j_{1}, \ldots, j_{k} \leqslant n \\
1 \leqslant i_{1}, \ldots, i_{k} \leqslant n}}(-1)^{k} \zeta_{i_{1} j_{1}} c_{i_{1} j_{2}} \zeta_{i_{2} j_{2}} c_{i_{2} j_{3}} \ldots \zeta_{i_{k} j_{k}} c_{i_{k} j_{1}} \\
&=(-1)^{k} \sum_{\substack{1 \leqslant i_{1}, j_{1} \leqslant n \\
=}} \zeta_{i_{1} j_{1}} q_{A}\left(i_{1}\right) C_{A}^{-t}\left(C_{A} C_{A}^{-t}\right)^{k-2} p_{A}\left(j_{1}\right)^{t} \\
&=(-1)^{k} \sum_{1 \leqslant v_{1}, \ldots, v_{k-1} \leqslant n}\left\langle q_{A}\left(v_{1}\right), p_{A}\left(v_{k-1}\right)\right\rangle\left\langle q_{A}\left(v_{2}\right), e_{A}\left(v_{1}\right)\right\rangle \ldots \\
&=\left\langle q_{A}\left(v_{k-2}\right), e_{A}\left(v_{k-3}\right)\right\rangle \operatorname{dim}_{A^{e}}(D A) C_{A^{e}}^{-t} e_{A^{e}}\left(v_{k-1}, v_{k-2}\right)^{t} \\
& \sum_{1 \leqslant v_{1}, \ldots, v_{k-1} \leqslant n}\left\langle q_{A}\left(v_{1}\right), p_{A}\left(v_{k-1}\right)\right\rangle\left\langle q_{A}\left(v_{2}\right), e_{A}\left(v_{1}\right)\right\rangle \ldots \\
&\left\langle q_{A}\left(v_{k-2}\right), e_{A}\left(v_{k-3}\right)\right\rangle\left\langle\operatorname{dim}_{A^{e}} D A, e_{A^{e}}\left(v_{k-1}, v_{k-2}\right)\right\rangle
\end{aligned}
$$




\section{References}

[1] A. BoldT, Methods to Determine Coxeter Polynomials, Linear Algebra and its Applications 230 (1995), pp 151154.

[2] W. GEIGLE and H. LENZING, A class of weighted projective curves arising in representation theory of finitedimensional algebras, in Singularities, Representation of Algebras, and Vector Bundles (Lambrecht, 1985), Lecture Notes Math. 1273 pp 265-297, Springer, Berlin (1987).

[3] D. Happel, On the Derived Category of a Finite-Dimensional Algebra, Comment. Math. Helv. 62 (1987), pp 339-389.

[4] D. HAPpel, The Trace of the Coxeter Matrix and Hochschild Cohomology, Linear Algebra and its Applications 258 (1997), pp 169-177.

[5] D. Happel, Hochschild Cohomology of Piecewise Hereditary Algebras, Colloquium Math. 78 (1998) no.2, pp 261-266.

[6] D. HAPPEL, A characterization of hereditary categories with tilting object. Invent. Math. 144 (2001), no. 2, pp 381-398.

[7] D. HAppel, The Coxeter Polynomial for a One Point Extension Algebra, J. Algebra 321 (2009), no. 7, pp 20282041.

[8] D. HAPPEL, Triangulated Categories in the Representation Theory of Finite Dimensional Algebras, LMS Lect Note Series 119 (1988).

[9] D. HAppel and I. ReITEN, Directing Objects in Hereditary Categories, Trends in the representation theory of finite-dimensional algebras, Contemp. Math. 229 (1998) pp 169-179.

[10] D. Happel, I. Reiten and S. Smalø, Piecewise hereditary algebras, Arch. Math. (Basel) 66 (1996), no. 3, pp 182-186.

[11] S. LACHE, Piecewise hereditary one point extensions, J. Algebra 226 (2000), no. 1, pp 53-70.

[12] S. LADKANI, On the periodicity of Coxeter transformations and the non-negativity of their Euler forms, Linear Algebra Appl. 428 (2008), no. 4, pp 742-753.

[13] P. LE MEUR, Topological invariants of piecewise hereditary algebras, arXiv:math/0702457.

[14] J. Lelong-FerRAnd and J-M. ARnAudiès, Cours de mathématiques, Tome 1, Algèbre, Troisième édition, 1er Cycle Universitaire, Classes Préparatoires, Mathématiques, Dunod, Paris (1977).

[15] P.A. MAcMahon, Combinatory Analysis. Two volumes (bound as one), Chelsea Publishing Co., New York (1960).

[16] C. RINGEL, Tame Algebras and Integral Quadratic Forms, Lecture Notes Math. 1099, Springer Verlag Berlin (1984).

[17] R. STEKOlSHCHIK, Notes on Coxeter Transformations and the McKay Correspondence, Springer Monographs in Mathematics, Springer-Verlag, Berlin, 2008.

MARCELO LANZILOTTA

Centro de Matemática (CMAT), Instituto de Matemática y Estadística Rafael Laguardia (IMERL), Universidad de la República, Iguá 4225, C.P. 11400, Montevideo, Uruguay.

E-mail address:marclan@cmat.edu.uy

MARIA JULIA REDONDO

Instituto de Matemática, Universidad Nacional del Sur, Av. Alem 1253, (8000) Bahía Blanca, Argentina.

E-mail address: mredondo@criba.edu.ar

RACHEL TAILLEFER (Corresponding author)

Laboratoire LaMUSE, Université de Saint-Etienne, Faculté des Sciences, 23, rue du Dr. P. Michelon, 42023 Saint-Etienne Cedex 2, France.

E-mail address: rachel.taillefer@univ-st-etienne.fr 